\title{
Dielectric Function of Nitride Semiconductors: Recent Experimental Results
}

\author{
R. GOLDHAHN* \\ Ilmenau Technical University, Institute of Physics \\ PF 100565, 98684 Ilmenau, Germany
}

\begin{abstract}
A review on the experimental determination of the dielectric function for hexagonal nitride semiconductors is presented. The peculiarities of nitride samples such as surface roughness and extended interface layers alter in comparison to an ideal film spectroscopic ellipsometry or reflectance spectra in a characteristic manner. It requires the application of multi-layer models for data analysis in order to determine reliable dielectric functions. Results of such an analysis for GaN covering a broad spectral range are given. Below the band gap, both ordinary and extraordinary components of the dielectric function tensor are determined for GaN as well as for AIN. The dielectric functions of MBE-grown In $\mathrm{N}$ characterised by a band gap of around $0.75 \mathrm{eV}$ and a sputtered film exhibiting an absorption edge of around $1.9 \mathrm{eV}$ are compared with results of first-principles calculations. Good agreement between theory and experiment is only found for the MBE-grown material providing further evidence that $\operatorname{InN}$ is a "narrow" band gap semiconductor. Finally, photocurrent measurements of a GaN Schottky-diode reveal the influence of electric fields on the shape of the excitonic absorption edge. The interpretation is supported by results of dielectric function calculations.
\end{abstract}

PACS numbers: 78.20.Ci, 78.40.Fy, 68.35.Ct, 71.35.Cc

\section{Introduction}

The nitride semiconductors such as $\mathrm{InN}$, GaN, and AIN have attracted considerable interest due to their outstanding physical, chemical, and mechanical properties, making them ideal candidates for the application in various devices. Light emitting diodes and laser diodes working in the green/blue to ultraviolet spectral regions [1] have been already commercialised, solar-blind photodetectors [2]

*e-mail: ruediger.goldhahn@tu-ilmenau.de 
are under intensive investigation. Transistors based on AlGaN/GaN heterostructures confining a polarisation induced two-dimensional electron gas allow high voltage, high power operation at microwave frequencies [3]. These devices have been realised by growing the thermodynamically stable hexagonal wurtzite $(\alpha-)$ phase. Compared to the $\alpha$-phase, only a few studies focus on the metastable cubic zinc-blende $(\beta-)$ modification. Only recently, electroluminescence of $\beta$-GaN was reported [4].

The binary compounds of both crystal structures, along with the related ternary alloys, represent a huge class of materials for which experimental determination of optical constants, i.e. complex dielectric function (DF) or complex refractive index, over an extended energy range and with the highest accuracy, is one of the major requirements for the understanding of basic physical properties. Let us consider $\alpha$-GaN for example. Studies in the infrared spectral region by reflectance/transmittance $(\mathrm{R}-\mathrm{T})$ spectroscopy or spectroscopic ellipsometry (SE) yield information on both lattice dynamics and free carrier absorption [5, 6]. Band-to-band transitions [7] and excitonic contributions [8] dominate the optical response in the visible/ultraviolet. A comparison of these data with electronic band-structure calculations provides an important test for the accuracy of the models $[7,8]$. Although the shape of the calculated spectra agrees already well with the experiments, the overall magnitude differs still appreciably [9].

Hexagonal InN provides another example for the necessity of such a comparison. It was a long time accepted that the band gap of this compound is around $1.89 \mathrm{eV}$ as determined from transmission measurements of sputtered films [10]. Recent investigations of films grown by molecular beam epitaxy (MBE) revealed a much lower absorption edge of $\sim 1 \mathrm{eV}$ [11]. The determination of the DF for both types of films over an extended energy range and a comparison with first-principles calculations provides a clear evidence that only the lower band gap material represents bulk-like InN [12]. A more detailed discussion will be given below.

Beside the fundamental aspects, precise knowledge of the DF, especially around and below the band gap, is of great technological relevance. It represents one of the essential quantities for the design and optimisation of the optical waveguide in separate-confinement heterostructure laser diodes [13] or for achieving highly reflective Bragg reflectors [14] used in vertical cavity surface emitting lasers. Furthermore, non-destructive optical methods can be developed for a fast monitoring of the thickness and properties of grown films [15] or the in situ control of the growth process [16].

Despite some recent achievements, the current knowledge of DF's for nitride semiconductors, even the binary compounds, is still rather poor [17]. It is mainly attributed to the fact that large bulk crystals with low defect and carrier concentrations are not available. Thus, almost all studies refer to layers grown heteroepitaxially by metalorganic chemical vapour deposition (MOCVD) or MBE on foreign non-lattice-matched substrates. Sapphire, 6H-SiC, Si(111), and GaAs(111) 
substrates are used for the deposition of the hexagonal films, while cubic phase nitrides have been reported on GaAs(001), 3C-SiC/Si(001) and $\mathrm{MgO}$. In order to achieve films of high crystalline quality, thin $(\sim 30 \mathrm{~nm}) \mathrm{GaN}$ or AlN buffer layers are grown at low temperatures (LT) prior the final deposition. It is obvious that the optical properties of those films differ from the ones of the subsequently grown top layer as demonstrated recently for $\alpha-\mathrm{GaN}$ [18] and $\beta-\mathrm{GaN}$ [19], respectively. If growth of a buffer layer is omitted, the formation of an amorphous layer [20] or void $[21,22]$ between the substrate and the active film is sometimes observed. All these peculiarities can be summarised under the concept interface layers. Finally, it should be noted that the non-ideal nitride surfaces influence the optical spectra. Surface roughness depends critically on the growth conditions, and organic/inorganic contamination or native oxides are found on air-exposed surfaces. Therefore, advanced models, taking into account both interface layers and surface contributions, have to be established for the experimental data analysis in order to get reliable DF's.

The present paper focus only on the hexagonal compounds. In the following sections, the basic physical quantities are defined and the fundamentals of the experimental methods including models for data analysis are discussed. Then, results of the DF determination for GaN are reported. Next, the anisotropy of GaN and AIN below the band gap is analysed. Then, the DF's of sputtered and MBE-grown InN films are presented and compared with theoretical results. In the final part, the influence of electric fields on the optical properties is discussed.

\section{Dielectric function and band structure}

\subsection{Fundamental relations}

The unstrained group-III nitrides with wurtzite structure belong to the $C_{6 v}^{4}\left(P 6_{3} m c\right)$ space group. Assuming that the optic axis ( $\boldsymbol{c}$-axis) is oriented normal to the surface ( $x-y$ plane) along the $z$ direction, which is found for most epitaxially grown films, the dielectric tensor of the hexagonal materials can be expressed by $[23]$

$$
\tilde{\varepsilon}=\left(\begin{array}{ccc}
\varepsilon_{x} & 0 & 0 \\
0 & \varepsilon_{y} & 0 \\
0 & 0 & \varepsilon_{z}
\end{array}\right)=\left(\begin{array}{ccc}
\varepsilon_{\mathrm{o}} & 0 & 0 \\
0 & \varepsilon_{\mathrm{o}} & 0 \\
0 & 0 & \varepsilon_{\mathrm{e}}
\end{array}\right) .
$$

The complex quantities $\varepsilon_{\mathrm{o}}$ (ordinary) and $\varepsilon_{\mathrm{e}}$ (extraordinary) are the principal components of the dielectric tensor and describe the response for light linear polarised perpendicular $(\boldsymbol{E} \perp \boldsymbol{c})$ and parallel $(\boldsymbol{E} \| \boldsymbol{c})$ to the optic axis, respectively. Their dependence on the photon energy $(\hbar \omega)$, known as complex dielectric function, is given in the following form:

$$
\varepsilon_{j}(\omega)=\varepsilon_{1, j}(\omega)+\mathrm{i} \varepsilon_{2, j}(\omega), \quad j=\mathrm{o}, \mathrm{e} .
$$

For each polarisation direction $j$, the real and imaginary parts of the DF obey the so-called Kramers-Kronig relation: 


$$
\varepsilon_{1}(\omega)=1+\frac{2}{\pi} P \int_{0}^{\infty} \frac{\omega^{\prime} \varepsilon_{2}\left(\omega^{\prime}\right) \mathrm{d} \omega^{\prime}}{\omega^{\prime 2}-\omega^{2}},
$$

where $P$ means the principal value of the integral. The complex refractive index $N_{j}$ (along the principal axis of the crystal), used instead in many publications, is related to $\varepsilon_{j}(\omega)$ via

$$
N_{j}(\omega)=n_{j}(\omega)+\mathrm{i} k_{j}(\omega)=\sqrt{\varepsilon_{j}(\omega)}, \quad j=\mathrm{o}, \mathrm{e} .
$$

Usually, the real part $n$ is likewise called refractive index, while the imaginary part $k$ is known as the extinction coefficient. From transmittance studies, however, only the absorption coefficient $\alpha(\omega)$ can be extracted which is related to $\varepsilon_{2}(\omega)$ via

$$
\alpha(\omega)=\frac{\omega}{n(\omega) c_{0}} \varepsilon_{2}(\hbar \omega)
$$

where $c_{0}$ denotes the velocity of light in vacuum.

\section{D. Theoretical approaches}

Although the present work is mainly concerned with the experimental determination of the DF, a few theoretical results illustrating the theoretical approach should be presented before. Christensen and Gorczyca calculated the band structures of the binary III-V nitrides in the framework of the local density approximation and derived from the one-particle wave functions the imaginary parts $\varepsilon_{2, \mathrm{o}}(\omega)$ and $\varepsilon_{2, \mathrm{e}}(\omega)$ for both polarisation directions [24]. Then, the corresponding $\varepsilon_{1}(\omega)$ values are obtained from $\varepsilon_{2}(\omega)$ by applying Eq. (3). In a detailed comparison with experimental results for wurtzite $\mathrm{GaN}$, Lambrecht et al. demonstrated the usefulness of this approach, especially, if the energies of the critical points (CP) of the band structure are considered $[7,25]$. However, they found an increasing overestimate of the theoretical intensity with increasing energy.

This problem can be solved if in addition the final state interaction between the excited electron and hole is taken into account as demonstrated recently by Benedict and Shirley [26]. Their calculated results for $\alpha$-GaN are summarised in Fig. 1. The full and dashed lines represent the ordinary and extraordinary components of the dielectric tensor, respectively. The data refer to zero temperature, and valence splitting at the $\Gamma$ point of the Brillouin zone was neglected (see below). Beside the band gap $\left(E_{0}\right)$ at $\sim 3.45 \mathrm{eV}, \varepsilon_{2}(\omega)$ shows pronounced features ( $E_{1}$ to $E_{3}$ ) above $6 \mathrm{eV}$ arising from transitions in the vicinity of the critical points. While all three contribute for $\boldsymbol{E} \perp \boldsymbol{c}, E_{2}$ should not be observable for the other polarisation direction. However, the transition probability around $E_{1}$ is strongly enhanced for $\boldsymbol{E} \| \boldsymbol{c}$. Therefore concerning the anisotropy in the low-energy range, $\varepsilon_{1, \mathrm{e}}(\omega)>\varepsilon_{1, \mathrm{o}}(\omega)$ is expected. A similar behaviour was calculated in Ref. [24]. Corresponding results for $\alpha-\mathrm{AlN}$ and $\alpha-\operatorname{InN}$ can be found in Refs. [9] and [27], respectively, as well as in Ref. [24].

The band structure calculations discussed above do not reveal the peculiarities in the gap energy range. The valence band maximum of the wurtzite nitrides is 

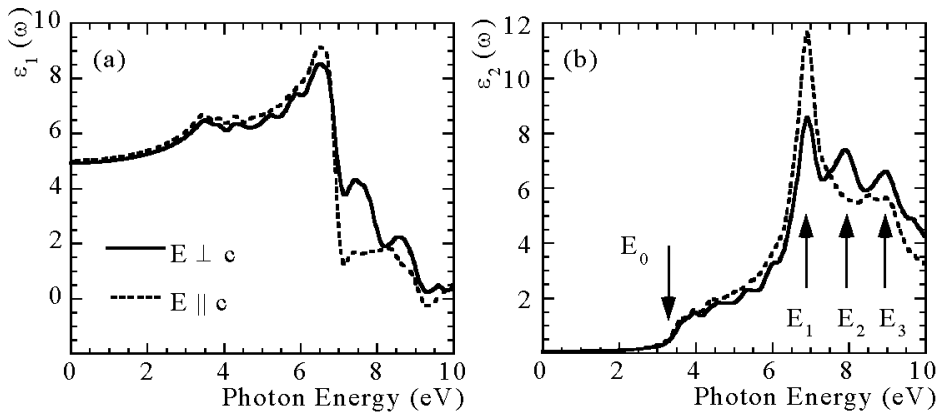

Fig. 1. Calculated real (a) and imaginary (b) part of the dielectric function for $\alpha$-GaN for $\boldsymbol{E} \perp \boldsymbol{c}$ and $\boldsymbol{E} \| \boldsymbol{c}$ (Data were taken from Ref. [26]). The arrows indicate critical points of the band structure.

located at the $\Gamma$ point of the Brillouin zone. Due to crystal-field and spin-orbit interaction it is split into three valence bands with $\Gamma_{9}^{\mathrm{v}}, \Gamma_{7}^{\mathrm{v}}$, and $\Gamma_{7}^{\mathrm{v}}$ symmetry $\left(\Gamma_{9}^{\mathrm{v}}\right.$ is the upper one for unstrained $\alpha$-GaN) [8]. The corresponding interband transitions to the lowest conduction band $\Gamma_{7}^{c}$ are usually denoted by $A, B$, and $C$, and the accompanying excitons by $F X^{A}, F X^{B}$, and $F X^{C}$, respectively. They dominate the optical response around the band gap. The $\Gamma_{9}^{\mathrm{v}}-\Gamma_{7}^{\mathrm{c}}$ transition (and $F X^{A}$ ) is only allowed for light polarisation $\boldsymbol{E} \perp \boldsymbol{c}$, while $\Gamma_{7}^{\mathrm{v}}-\Gamma_{7}^{\mathrm{c}}$ (and $F X^{B}$ and $F X^{C}$ ) transitions contribute for both polarisation directions.

Kornitzer et al. [28] studied nearly strain-free homoepitaxial $\alpha$-GaN layers by means of reflectance spectroscopy $(\boldsymbol{E} \perp \boldsymbol{c})$ at helium temperature and determined the $A-B$ and $B-C$ energetic splitting with $4.6 \mathrm{meV}$ and $16.9 \mathrm{meV}$, respectively. If instead heteroepitaxial layers are studied, all quantities such as e.g. band-to-band and excitonic transition energies as well as their oscillator strengths depend sensitively on residual strain of the investigated layers. Consequently, they depend on the difference of the lattice constants and thermal expansion coefficients between layer and substrate, substrate orientation, layer thickness, buffer layer growth, etc. It can alter the selection rules, given above, under certain conditions (for a detailed analysis see e.g. Ref. [8]).

\section{Experimental methods and data analysis}

\subsection{3-phase model}

Polarised reflectance and spectroscopic ellipsometry are the most powerful experimental methods for studying the dielectric function and band structure of semiconductors. Both techniques are based on the determination of the elements of the Jones $2 \times 2$ complex-amplitude reflection matrix [29]

$$
\left[\begin{array}{c}
E_{\mathrm{r} p} \\
E_{\mathrm{r} s}
\end{array}\right]=\left[\begin{array}{cc}
r_{p p} & r_{p s} \\
r_{s p} & r_{s s}
\end{array}\right]\left[\begin{array}{c}
E_{\mathrm{i} p} \\
E_{\mathrm{i} s}
\end{array}\right]
$$


connecting the incident $\left(E_{\mathrm{i}}\right)$ and reflected $\left(E_{\mathrm{r}}\right)$ electric fields for $s$ - and $p$-polarisation. Allowing data analysis for any arbitrary sample geometry (e.g. hexagonal film on an anisotropic off-oriented substrate), the most general unified approach to compute the reflection matrix, characterising the sample properties, is the $4 \times 4$ matrix method [29, 30].

Since it is far beyond the scope of this paper to discuss all possible layer geometries, only the simplest case, known as the 3-phase model, should be briefly discussed. It consists of a planar hexagonal nitride film of thickness $d$ with ordinary and extraordinary DF's $\varepsilon_{\mathrm{o}}$ and $\varepsilon_{\mathrm{e}}$, respectively, on a substrate $\left(\varepsilon_{\mathrm{s}}\right)$ in non-absorbing ambient $\left(\varepsilon_{\mathrm{a}}\right)$. The optical axis is oriented normal to the ambient-sample boundary corresponding to most experimental studies. In this case, the off-diagonal elements of the reflection matrix vanish $\left(r_{p s}=r_{s p}=0\right)$, while the diagonal reflection coefficients are given by

$$
\begin{aligned}
& r_{p p}=\frac{r_{01, p p}+r_{12, p p} \exp \left(\mathrm{i} 2 \beta_{p}\right)}{1+r_{01, p p} r_{12, p p} \exp \left(\mathrm{i} 2 \beta_{p}\right)}, \\
& r_{s s}=\frac{r_{01, s s}+r_{12, s s} \exp \left(\mathrm{i} 2 \beta_{s}\right)}{1+r_{01, s s} r_{12, s s} \exp \left(\mathrm{i} 2 \beta_{s}\right)} .
\end{aligned}
$$

In Eqs. (7) and (8), $r_{01, p p}, r_{12, p p}$ and $r_{01, s s}, r_{12, s s}$ represent the Fresnel coefficients for the reflection of $p$ - and $s$-polarised light at the ambient-film (01) and film-substrate interface (12), respectively. If the layers are deposited on uniaxial substrates such as sapphire or $6 \mathrm{H}-\mathrm{SiC}$, the anisotropy of the substrate has to be taken into account in addition which can be found in Ref. [29]. The phase shifts $\beta_{p}$ and $\beta_{s}$ are given by

$$
\begin{aligned}
& \beta_{p}=2 \pi \frac{d}{\lambda}\left(\varepsilon_{\mathrm{o}}-\frac{\varepsilon_{\mathrm{o}}}{\varepsilon_{\mathrm{e}}} \varepsilon_{\mathrm{a}} \sin ^{2} \Phi\right)^{1 / 2}, \\
& \beta_{s}=2 \pi \frac{d}{\lambda}\left(\varepsilon_{\mathrm{o}}-\varepsilon_{\mathrm{a}} \sin ^{2} \Phi\right)^{1 / 2},
\end{aligned}
$$

where $\lambda$ is the wavelength of light in vacuum, $\Phi$ denotes the angle of incidence in the ambient. From the equations given above, the following characteristic properties should be noted:

(i) the optical response for $s$-polarized light depends only on the ordinary DF of the film,

(ii) under oblique angle of incidence, all measurements using $p$-polarized light depend on both, $\varepsilon_{\mathrm{o}}$ and $\varepsilon_{\mathrm{e}}$,

(iii) for investigations above the band gap, Eqs. (7) and (8) reduce to $r_{p p}=$ $r_{01, p p}$ and $r_{s s}=r_{01, s s}$, if the layer thickness is much larger than the light penetration depth. 


\subsection{Reflectance measurements}

In reflectance studies, the quantities

$$
R_{p}=\left|r_{p p}\right|^{2} \quad \text { and } \quad R_{s}=\left|r_{s s}\right|^{2}
$$

are determined as a function of photon energy and angle of incidence. However, in most studies $\Phi=0$ is chosen $\left(R_{p}=R_{s}=R\right)$ which should be discussed at first. Two cases have to be distinguished, studies either above or below the band gap. In the former one, a thick smooth and clean nitride film represents a semi-infinite substrate, and the 3-phase model reduces to the so-called 2-phase model (ambient/substrate). Generally, in order to allow for the determination of both the real and imaginary parts of the DF, measurements of $R$ must cover a broad spectral range (up to photon energies deep in the vacuum-ultraviolet) followed by Kramers-Kronig transformation. If the $\boldsymbol{c}$-axis lies in the surface plane, both polarisation directions can be studied (either $\boldsymbol{E} \perp \boldsymbol{c}$ or $\boldsymbol{E} \| \boldsymbol{c}$ ). This configuration was used by Dingle et al. for revealing the symmetry of the three uppermost valence bands of $\alpha$-GaN [31], however, DF's were not reported. A synchrotron radiation reflectance study up to $30 \mathrm{eV}$ for wurtzite $\mathrm{GaN}$ with normal c-axis orientation was reported in Ref. [7]. While the energetic positions of the CPs are already in good agreement with calculations, the experimental magnitudes are much too low, especially at higher photon energies. It turns out that the deviations are caused by the peculiarities of the surface as demonstrated below.

Most reflectance studies of nitride films, however, are devoted to the determination of the real part of the ordinary DF below the band gap and refer to $\Phi=0$ with the $\boldsymbol{c}$-axis being normal to the surface. This special case should be discussed in more detail. Let us assume a smooth $\alpha$-GaN film $(1.6 \mu \mathrm{m})$ on GaAs substrate without an interlayer. Applying the 3-phase model, a reflectance spectrum is calculated as shown by the solid line in Fig. 2. The reflectance of the bare substrate (dashed line) and the envelope of the interference extrema (dotted lines) are given

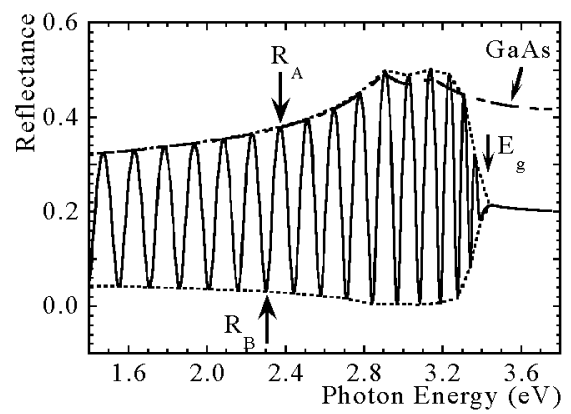

Fig. 2. Calculated reflectance (3-phase model) for a $1.6 \mu \mathrm{m}$ thick $\alpha$-GaN film on GaAs substrate (solid line). The dashed and dotted lines represent the reflectance of the substrate and the envelope of the interference extrema, respectively. 
for comparison. The following characteristic properties should be noted. First, the envelopes are independent of the layer thickness. Second, below $2.8 \mathrm{eV}$, where the substrate is weakly absorbing, the envelope of the maxima coincides with the reflectance of GaAs $\left(R_{A}\right)$. This can serve as a test for the applicability of the 3-phase model for the analysis of experimental data as we will see later. Third, the only information regarding the dispersion and absolute value of the DF $\varepsilon_{1,0}(\omega)$ for the $\alpha$-GaN film can be obtained by analysing the spacing between the oscillations and the height of the minima $\left(R_{B}\right)$, respectively. These distinguishing features validate as long as the optical constants of the film are lower than substrate ones (e.g. GaAs, $6 \mathrm{H}-\mathrm{SiC}$, or $\mathrm{Si}$ ). For sapphire with a lower refractive index, only the meaning of $R_{A}$ have to be exchanged for $R_{B}$ and vice versa. If reflectance data are taken at oblique incidence, then, according to Eqs. (9) and (10), $s$ - and $p$-polarised light undergo different phase changes. The effect can be used to determine $\varepsilon_{1, e}(\omega)$ in addition [32].

\subsection{Spectroscopic ellipsometry}

Spectroscopic ellipsometry analyses the change in the polarisation state of light upon interaction with a sample [29]. A monochromatic, linearly polarised electromagnetic plane wave is reflected under non-normal incidence. While the vector components $E_{\mathrm{i} p}$ and $E_{\mathrm{i} s}$ of the incident field are in phase, the reflected components $E_{\mathrm{r} p}$ and $E_{\mathrm{r} s}$ show a phase difference $\Delta$, i.e. the reflected wave is elliptically polarised. Furthermore, the ratio of the magnitudes has changed. Both effects can be expressed via the complex ratio $\rho$ of the reflection coefficients by

$$
\rho=\frac{r_{p p}}{r_{s s}}=\tan \Psi \exp (\mathrm{i} \Delta) .
$$

In standard variable angle SE, the ellipsometric parameters $\Psi$ and $\Delta$ are analysed at each photon energy. Because SE studies are performed with the intent of determining optical constants, the ratio $\rho$ is often converted to the so-called pseudo-dielectric function $\langle\varepsilon\rangle$ by means of the isotropic 2-phase model (ambient/substrate):

$$
\langle\varepsilon\rangle=\left\langle\varepsilon_{1}\right\rangle+\mathrm{i}\left\langle\varepsilon_{2}\right\rangle=\sin ^{2} \Phi\left[1+\tan ^{2} \Phi\left(\frac{1-\rho}{1+\rho}\right)^{2}\right] .
$$

It should be emphasised that $\langle\varepsilon\rangle$ represents normally rather sample properties than material properties. Only in the limiting case of a thick isotropic film (investigated above the band gap) without any surface roughness, oxide layers, and contamination etc., the quantity $\langle\varepsilon\rangle$ obtained from Eq. (13) is identical to the intrinsic dielectric function $\varepsilon$ of the layer. Hence, all other cases require the drawing up of appropriate models for fitting $\Psi$ and $\Delta$.

Above the band gap of the hexagonal compounds at least four quantities $\left(\varepsilon_{1, \mathrm{o}}, \varepsilon_{2, \mathrm{o}}, \varepsilon_{1, \mathrm{e}}, \varepsilon_{2, \mathrm{e}}\right)$ are unknown, but $\Psi$ and $\Delta$ depend mainly on the in-plane tensor components [33]. Therefore, using layers with $\boldsymbol{c}$-axis orientation normal to 
the surface, $\varepsilon_{\mathrm{e}}$ cannot be determined. A more promising way would be the investigation of samples for which the optical axis is significantly off-normal. In this case the off-diagonal elements of the reflection matrix $r_{s p}$ and $r_{p s}$ are different from zero and can be detected by SE. Preliminary results of those investigations employing $\boldsymbol{M}$-plane $\mathrm{Al}_{0.1} \mathrm{Ga}_{0.9} \mathrm{~N}(1 \overline{1} 00)$ grown on $\gamma$-LiAlO ${ }_{2}(100)$ have been reported recently [34].

\subsection{Influence of non-ideal surfaces and interfaces}

In the previous section, fundamentals of data analysis were presented assuming an ideal single layer on a substrate or crystals in order to determine the DF. However in reality nitride films show some peculiarities requiring a more elaborate strategy. Optical spectra of nitrides may be essentially influenced by surface effects, such as oxide layers, roughness and contamination, or non-ideal film-substrate interfaces caused by e.g. buffer layers, inhomogeneous strain relaxation or deteriorated substrate surfaces. In the following, it is demonstrated how to prove these effects and how to take them into account in data analysis in order to obtain more reliable DF's.

\subsubsection{Surface contamination and oxides}

Edwards et al. [35] observed essential changes in the pseudo-DF of hexagonal GaN, AlN, and AlGaN due to wet chemical treatments in real time. The layers were grown by MOCVD on vicinal $6 \mathrm{H}-\mathrm{SiC}$ substrates. The behaviour of $\langle\varepsilon\rangle$ for GaN for example was studied after treatment in methanol $(\mathrm{MeOH}), \mathrm{NH}_{4} \mathrm{OH}, \mathrm{NaOH}$, and $0.01 \% \mathrm{Br}$ in methanol in the energy range up to $5.5 \mathrm{eV}$. The general trend can be summarised as follows: after each cleaning step $\left\langle\varepsilon_{1}\right\rangle$ increased while $\left\langle\varepsilon_{2}\right\rangle$ decreased which is explained by a removal of an overlayer typically found on air-exposed surfaces. The analysis evidenced that half of the removed overlayer was soluble in $\mathrm{MeOH}$ and $\mathrm{H}_{2} \mathrm{O}$, and, therefore, is related to organic and inorganic contamination. The remaining part of the overlayer was presumably a $\mathrm{Ga}_{2} \mathrm{O}_{3}$-like oxide.

The influence of a $\mathrm{Ga}_{2} \mathrm{O}_{3}$-like oxide is even more dramatic at higher energies. This was revealed by Wethkamp [36] by measuring the pseudo-DF of a $\alpha$-GaN
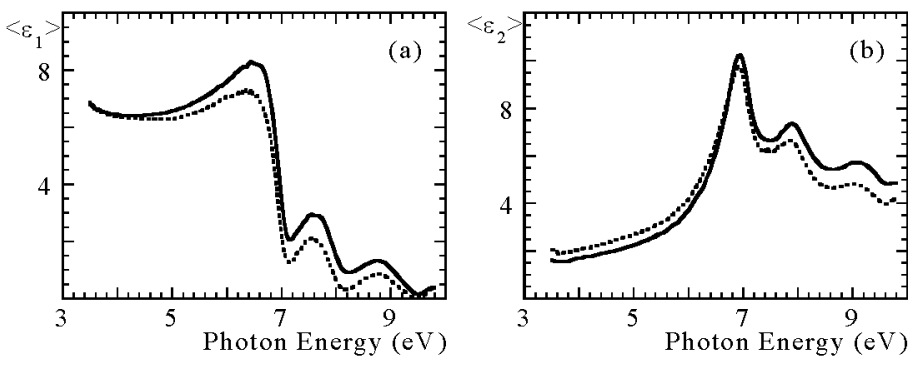

Fig. 3. Real $\left\langle\varepsilon_{1}\right\rangle$ (a) and imaginary $\left\langle\varepsilon_{2}\right\rangle$ (b) part of the pseudo-dielectric function of $\alpha$-GaN before (dashed lines) and after oxide removal (full lines) by thermal desorption in $\mathrm{UHV}$ at $500^{\circ} \mathrm{C}$ for 10 minutes (Data were taken from Ref. [36]). 
sample grown by MOCVD on sapphire before and after thermal desorption at $\sim 500^{\circ} \mathrm{C}$ in ultra-high vacuum. The change of the data shown in Fig. 3 can be explained by a removal of $\sim 1 \mathrm{~nm}$ thick oxide overlayer. Note that at higher photon energies both the real and imaginary parts of the pseudo-DF increased after treatment indicating the absorption due to the oxide. However, this surface cleaning procedure deteriorated the surface flatness. The rms roughness $\delta$ increased from $0.28 \mathrm{~nm}$ to $0.87 \mathrm{~nm}$ which was determined from atomic microscopy studies (AFM).

Both studies demonstrated the extremely high sensitivity of SE to thin overlayers. The method can be used to assess the preparation of chemically clean abrupt nitride surfaces. A more detailed review of $e x$ situ and $i n$ situ cleaning procedures for preparing device-quality AlN and GaN surfaces was presented in Ref. [37].

\subsubsection{Surface roughness}

The disturbing influence of surface contamination or oxides can be avoided by a proper surface pre-treatment. But even then, reflectance and SE studies suffer from the contribution caused by surface roughness. Despite a large number of reports on optical spectra of nitrides, there are only a few works for $\alpha$-GaN $[15,17$, $22,38,39]$, where the influence of the surface roughness was systematically investigated. Kawashima et al. [39] reported SE spectra for two $\alpha$-GaN films grown by MOCVD on sapphire. The films exhibited the rms surface roughness of $0.18 \mathrm{~nm}$ and $5.2 \mathrm{~nm}$ leading to essential differences in the pseudo-DF above the band gap. At a photon energy of $3.8 \mathrm{eV}$, values of $\left\langle\varepsilon_{1}\right\rangle=6.71$ and $\left\langle\varepsilon_{2}\right\rangle=1.80$ as well as $\left\langle\varepsilon_{1}\right\rangle=6.32$ and $\left\langle\varepsilon_{2}\right\rangle=2.28$ were determined for the smoother and rougher sample, respectively. Hence, it can be concluded that $\left\langle\varepsilon_{2}\right\rangle$ increases with roughness, while $\left\langle\varepsilon_{1}\right\rangle$ decreases. This behaviour is similar to the case of surface contamination/oxides.

The above discussed experimental results demonstrated the strong influence of surface roughness on the pseudo-DF. However, because we are interested in determining a real DF for each material, we should know how to model this effect for the analysis of SE and reflectance spectra. Two different approaches in attempting to solve this problem are presented and compared to each other.

Starting from hypothesis concerning a transition layer on an isotropic substrate surface, Sivukhin [40] proposed a model in order to explain elliptic polarisation of reflected light (for a review see Ref. [41] and references therein). Under influence of the incident wave, the atoms/molecules of the substrate acquire dipole moments and produce secondary waves. The reflected light is the result of interference of the secondary waves. From physical grounds, one can believe that the polarisability of "surface" atoms/molecules differs from that of atoms/molecules in a bulk, and therefore, two types of secondary radiation sources, surface sources and volume sources, can be considered. Since experimentally one measures the field at distances far away from the substrate compared with atomic and interatomic 
dimensions, the effect of the discrete surface sources can be approximated by continuously distributed sources, i.e., a flat-parallel layer of a polarisable effective medium with optical properties different from those of the substrate material.

This model is intensively used in ellipsometric studies to simulate roughness. If the mean height and correlation length of the irregularities on a rough surface are both much less than the wavelength of light, multiple-scattering depolarisation is not significant and the contribution of the polarisation of the rough surface to the far-field radiation pattern can be approximated by one or more layers (overlayers) of polarisable effective media sandwiched between a perfect substrate and a perfect ambient [41]. Usually, a rough overlayer is considered as a mixture of the ambient and the substrate with optical constants calculated using one of the effective medium approximation (EMA) models discussed in detail in Refs. [41] and [42]. The simplest among them is a linear model in which the effective complex DF of a composed medium is postulated to be a linear function of volume fractions of constituents. More common are the Maxwell-Garnett [43] and the Bruggeman [44] EMA's.

The second approach considers scattering of light by surface irregularities in reflectance studies, i.e. after reflection from a rough surface, not only specularly reflected waves occur but a part of the light is scattered into other directions. The scattered light is not detected and hence the measured reflectance $(R)$ of a rough opaque substrate is smaller than that $\left(R_{s}\right)$ of a smooth substrate. Calculations under general assumptions about the shape of surface irregularities lead to a relation $[45,46]$

$$
R=R_{s} \exp \left(-\frac{16 \pi^{2} n_{\mathrm{a}}^{2} \delta^{2}}{\lambda^{2}}\right)
$$

where $n_{\mathrm{a}}$ is the refractive index of an ambient. The formula allows the correction of experimental $R$-spectra in order to get $R_{s}$ once knowing $\delta$ for example from AFM measurements. Applications to nitride semiconductors demonstrating the usefulness of this approach and its accuracy were reported recently [22, 38]. A more detailed comparison of both models can be found in Ref. [17].

\subsubsection{Interlayers and non-abrupt internal boundaries}

There is a large number of reflectance, transmittance or SE investigations dedicated to the determination of optical constants below the band gap of the nitrides. Even for the most intensively studied material $\alpha-\mathrm{GaN}$, the reported refractive indices and the energy dispersion $(\mathrm{d} n / \mathrm{d} E)$ deviate from each other up to $6 \%$ and an order of magnitude, respectively [15]. One reason for the differences might be that data analysis is carried out in most works using the simplest 3-phase model (ambient/film/substrate) only. It neglects all non-idealities in the film/substrate interface region introduced by the growth process. Non-ideality means the presence of interlayers with optical constants being different from the ones of the substrate and film, e.g. an amorphous layers [20] or void formation at the substrate sur- 
face $[21,22]$ as evidenced by transmission electron microscopy (TEM) studies. A high density of stacking faults is typically found in the low-temperature $\alpha$-GaN nucleation layer grown on sapphire [47]. Finally, Micro-Raman studies on thick $\alpha$-GaN layers grown on sapphire or $6 \mathrm{H}-\mathrm{SiC}$ revealed an inhomogeneous strain relaxation in the growth direction [48]. All these effects alter the optical properties, and therefore, such a sample represents a more complicated multi-layer system.

Reflectance measurements over a broad spectral range and the comparison with the expected behaviour within the 3 -phase model provide a sensitive technique to prove the presence of interlayers and to draw conclusions concerning the interface optical properties [15]. Two representative examples should be discussed in detail [49]. Hexagonal GaN films were grown on GaAs(111)B substrates by MBE using a rf activated plasma source to provide atomic nitrogen and an elemental source for $\mathrm{Ga}$. Oxide removal from substrate surface was carried out $620^{\circ} \mathrm{C}$ under As flux. Then, for one sample a low temperature nucleation layer was generated at $610^{\circ} \mathrm{C}$ by 10 min nitridation of the substrate. The other sample was directly heated to the deposition temperature of $710^{\circ} \mathrm{C}$. Both samples were grown under $\mathrm{N}$-rich conditions with an identical $\mathrm{N} / \mathrm{Ga}$ ratio up to a final layer thickness of approximately $1 \mu \mathrm{m}$.
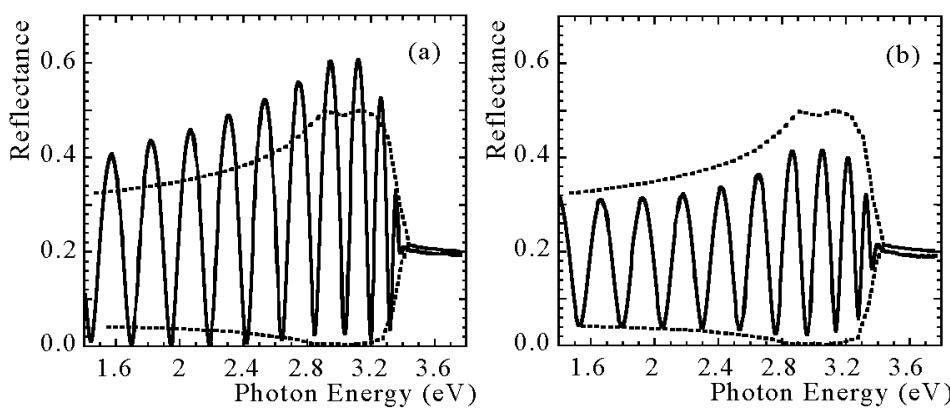

Fig. 4. Experimental reflectance spectra (solid lines) for two $\alpha$-GaN films grown by MBE on $\mathrm{GaAs}(111) \mathrm{B}$ substrates either without nucleation layer (a) or with 10 minutes nitridation of the substrates prior subsequent growth (b). The calculated envelopes of Fig. 2 are shown for comparison.

The solid lines in Fig. 4a and b represent the reflectance of the two samples, and the envelopes (dashed lines) correspond to Fig. 2. Characteristic deviations to the simple 3 -phase model are observed over the whole range. The difference between the experimental curves and the envelope above the band gap is caused by surface roughness. Below the gap neither for the directly grown sample nor the nitridated sample the reflectance maxima coincide with the envelope. This observation represents the direct experimental proof of an interlayer requiring a multi-layer approach for the data analysis. As demonstrated in Ref. [15], an enhanced reflectance for the maxima (Fig. 4a) indicates an extended interface 
layer with a DF ( $\left.\varepsilon_{\text {int }}\right)$ being lower than for the subsequently grown bulk-like GaN film $\left(\varepsilon_{\mathrm{GaN}}\right)$, while the condition $\varepsilon_{\mathrm{GaN}}<\varepsilon_{\mathrm{int}}<\varepsilon_{\mathrm{GaAs}}$ leads to a reduced reflectance (Fig. 4b). Without going into detail, the main reason for the extended interface in the present case is the formation of pits (voids) at the substrate surface during the growth process. The deteriorated substrate layer can be represented by an EMA film (GaAs with voids), treating its thickness $d_{\text {int }}$ and the void fraction $f_{\text {int }}$ as adjustable parameters. A complete data analysis is reported in Ref. [49]. The major result is that the nitridation process reduces strongly the void formation and the size of the interface region. Figure $4 \mathrm{a}$ corresponds to $f_{\text {int }}=0.64$, while $f_{\text {int }}=0.15$ is found for Fig. $4 \mathrm{~b}$.

A more common substrate for $\alpha$-GaN growth is $6 \mathrm{H}-\mathrm{SiC}$. In Ref. [38] a single crystalline film grown by MBE using a low-temperature GaN buffer layer was investigated. No evidence was found for either extended void formation or amorphous layers by cross-sectional and high-resolution TEM studies. However, within $30-50 \mathrm{~nm}$ away from the substrate/film boundary, the measurements revealed a clearly visible strain contrast and a high density of threading dislocations of mixed character which self-annihilate and decrease in intensity in the depth of the film. Although this disturbed region near the boundary might exhibit a refractive index only slightly different to GaN, it is unambiguously verified by reflectance studies.

\section{Experimental results and discussion}

\subsection{Dielectric function of hexagonal GaN}

Hexagonal GaN represents the most intensively studied nitride compound. But it turns out that even for this material the components of the dielectric tensor are not known with sufficient accuracy over a broad spectral range. For investigations above the band gap only pseudo-dielectric functions were reported in most cases, i.e. those data suffer from the influence of the surface peculiarities. Wethkamp compared the $\langle\varepsilon\rangle$ 's of various MBE- and MOVPE-grown films on sapphire and $6 \mathrm{H}-\mathrm{SiC}$ substrates with bulk $\mathrm{GaN}$ crystals as obtained from $\mathrm{SE}$ measurements [36]. The sample with the best crystalline quality and an electron concentration as low as $5 \times 10^{15} \mathrm{~cm}^{-3}$ which was grown by MOVPE on sapphire substrate yielded already prior thermal desorption of the oxide the largest $\left\langle\varepsilon_{2}\right\rangle$ value at around $6.8 \mathrm{eV}$, but even a larger value after treatment (Fig. 2).

Taking these data and correcting them numerically for the surface roughness, one obtains the spectral dependence of the DF as shown in Fig. 5 by the circles. Because experimental data refer to an angle of incidence of $\Phi=67.5^{\circ}$ and therefore to an angle of refraction of $\sim 20^{\circ}$ they correspond nearly to the ordinary DF. The assumption is corroborated by the fact that all three critical points of the band ( $E_{1}, E_{2}$, and $E_{3}$ ) are strongly pronounced while $E_{2}$ should be missing for $\boldsymbol{E} \| \boldsymbol{c}$. Hence, the theoretical results for the perpendicular polarisation direction as already shown in Fig. 1 are plotted for comparison (dashed lines). Concerning the 

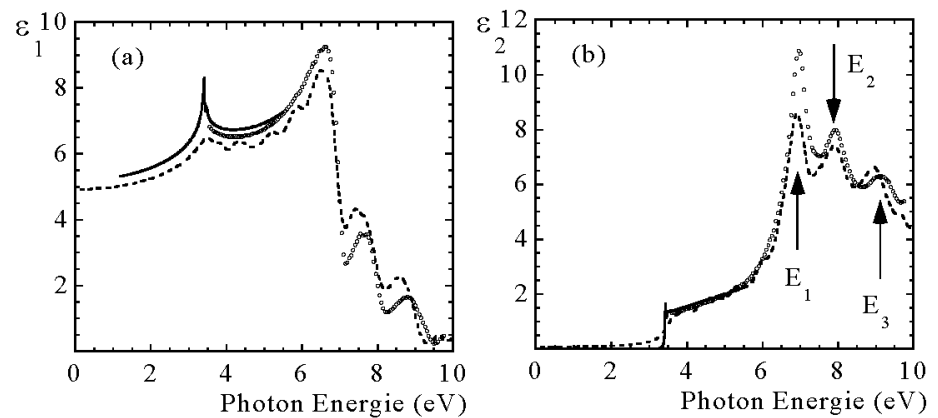

Fig. 5. Real (a) and imaginary (b) part of the dielectric function for hexagonal GaN. The open circles are obtained from Fig. 3 after correcting for roughness. Theoretical results of Fig. 1 for $\boldsymbol{E} \perp \boldsymbol{c}$ are shown for comparison (dashed line).

energetic positions already excellent agreement is obtained. However, the experimental magnitude is larger than calculated, especially in the range of $E_{1}$. There are two possible explanations. Figure 1 indicated a larger transition probability for light polarisation parallel to the $\boldsymbol{c}$-axis around $E_{1}$. Therefore, an averaged experimental DF with increasing $\varepsilon_{\mathrm{e}}$ contribution (corresponding to the polarisation ratio) shows as well a larger peak value than $\varepsilon_{0}$ alone. Secondly, the calculated values for $\varepsilon_{0}$ are still too low. Experimental results concerning the anisotropy as discussed in the next section show that in contrast to the calculations $\varepsilon_{1}$ is never less than 5 in the low-energy range neither for the ordinary nor the extraordinary component.

Let us turn to the energy range around the band gap of $\sim 3.45 \mathrm{eV}$. The full lines in Fig. 5 represent fitted data for a $1800 \mathrm{~nm}$ thick GaN film grown by MOVPE on sapphire substrate with an low-In content interface layer. The surface roughness was $\delta=0.3 \mathrm{~nm}$. XRD measurements yielded lattice constants of $c=5.190 \AA$ and $a=3.182 \AA$. As it can be seen in the graph these new data match nearly perfectly with the studies of Ref. [36]. In comparison to our previous investigation of a MBE-grown GaN film on $6 \mathrm{H}-\mathrm{SiC}$ [38] the features around the band gap are

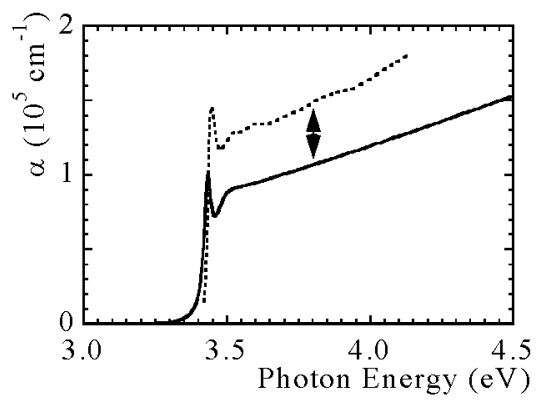

Fig. 6. Absorption coefficient of $\alpha-\mathrm{GaN}$ as calculated from the full lines of Fig. 5. The film was deposited on sapphire substrate. The dashed line refers to SE investigations without taking into account roughness [50]. Note the difference in the absolute values. 
much sharper emphasising the superior optical properties of the MOVPE film. From the $\varepsilon_{1}$ and $\varepsilon_{2}$ values the absorption coefficient $\alpha$ can be calculated which is shown by the full line in Fig. 6 . The peak at $3.433 \mathrm{eV}$ is attributed to the superposition of the free excitonic $F X^{A}$ and $F X^{B}$ transitions. But Fig. 6 emphasises as well the necessity of SE data analysis concerning surface properties. Although the qualitative shape of $\alpha$ is maintained if pseudo-DF's are used (dashed line) the absolute value is much larger [50] with the consequence that the light penetration depth would be underestimated.

What remains is the question concerning the anisotropy in the high-energy range. To the best of our knowledge now data were reported so far for GaN. However, preliminary results of pseudo-DF's for $\boldsymbol{M}$-plane $\mathrm{Al}_{0.1} \mathrm{Ga}_{0.9} \mathrm{~N}(1 \overline{1} 00)$ with the $\boldsymbol{c}$-axis orientation in the surface plane evidence indeed that the $E_{2}$ critical point is not observed for $\boldsymbol{E} \| \boldsymbol{c}[34]$.

\subsection{Optical anisotropy of $\alpha-G a N$ and $\alpha-A l N$ below the band gap}

Although the anisotropy of $\varepsilon_{1}$ below the band gap $\left(\varepsilon_{2}=0\right)$ is much lower than in the high-energy range it is easier to access. Two techniques are used. Fundamentals of the prism coupling technique (PCT) are found in Ref. [51]. This method is based on the waveguiding properties of the film. However, PCT is restricted to photon energies of available lasers and is applicable only to transparent films grown on substrates with lower refractive index (e.g. sapphire) due to the necessary waveguide arrangement. In fact, these limitations do not occur in reflectance and SE studies. According to Eqs. (9) and (10), the $p$ - and $s$-polarised waves undergo different phase shifts for non-normal incidence of light because $\varepsilon_{\mathrm{e}}$ enters $\beta_{p}$ but not $\beta_{s}$. Therefore, the extrema of the interference oscillations in polarised reflectance measurements (detection of $R_{p}$ and $R_{s}$ ) show a different energy shift while changing the angle of incidence. The analysis yields $\varepsilon_{1, \mathrm{o}}$ and $\varepsilon_{1, \mathrm{e}}$ as demonstrated in Ref. [32] for GaN.

In comparison to reflectance studies, SE provides an even higher sensitivity as shown recently by us [52]. We fitted the data for GaN and AlN films on $6 \mathrm{H}-\mathrm{SiC}$ substrates taken at various angles of incidence at each photon energy. The spectral dependences of the DF's can be modelled by an analytic formula. It becomes clear from Fig. 1 that the anisotropy and the dispersion below the band gap is originate from two contribution, one arises from the band gap, but the second one is due to the large oscillator strength around the $E_{1}$ critical point. Taking both contributions into account the spectral dependence of real part of $\varepsilon$ can be described by

$$
\varepsilon_{1}(\hbar \omega)=1+\frac{2}{\pi}\left(\frac{A_{0}}{2} \ln \frac{E_{1}^{2}-(\hbar \omega)^{2}}{E_{0}^{2}-(\hbar \omega)^{2}}+\frac{A_{1} E_{1}}{E_{1}^{2}-(\hbar \omega)^{2}}\right)
$$

with the adjustable parameters $E_{0}, E_{1}, A_{0}$, and $A_{1}$ for the corresponding polarisation directions. Their values are summarised in Table. It should be noted that the energies do not fit exactly to the critical point energies. A more detailed discussion is given in Ref. [52]. 
TABLE

Parameters of Eq. (15) for the real part $\varepsilon_{1}$ of the ordinary (o) and extraordinary (e) dielectric functions of wurtzite AlN and GaN in the transparent region.

\begin{tabular}{c|c|c|c|c|c}
\hline \hline Material & $\mathrm{DF}$ & $A_{0}$ & $E_{0}[\mathrm{eV}]$ & $A_{1}[\mathrm{eV}]$ & $E_{1}[\mathrm{eV}]$ \\
\hline$\alpha$-AlN & $\varepsilon_{1, \mathrm{o}}$ & 4.664 & 6.465 & 27.14 & 10.81 \\
& $\varepsilon_{1, \mathrm{e}}$ & 4.944 & 6.398 & 30.28 & 9.461 \\
\hline$\alpha$-GaN & $\varepsilon_{1, \mathrm{o}}$ & 1.837 & 3.450 & 40.65 & 8.175 \\
& $\varepsilon_{1, \mathrm{e}}$ & 1.929 & 3.504 & 41.89 & 8.164
\end{tabular}

The full and dashed lines in Fig. 7 represent these SE results for $\varepsilon_{0}$ and $\varepsilon_{e}$, respectively, as well as previously published data for GaN extracted from PCT [51] and AlN obtained from reflectance studies of bulk crystals [53] (open symbols refer to the extraordinary part). In agreement with calculations the extraordinary component is larger than the ordinary one for both materials over the considered spectral range. If an isotropic model would be used to analyse SE data then an a veraged $D F$ is determined. Those data lie even slightly below $\varepsilon_{1, o}$ and not between $\varepsilon_{1, \circ}$ and $\varepsilon_{1, \mathrm{e}}$ as one could expect.

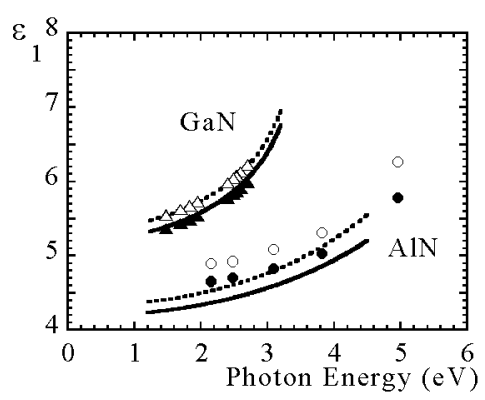

Fig. 7. Real part of the ordinary (full lines, filled symbols) and extraordinary DF (dashed lines, open symbols) DF. The upper (lower) curves refer to $\alpha$-GaN $(\alpha$-AlN). Corresponding data indicated by triangles and circles were reported in Refs. [51] and [53], respectively.

The representation by analytic formula allows extrapolation to zero photon energy yielding the high-frequency dielectric constant $\varepsilon(\infty)$ used to describe the interband contribution while fitting infrared spectra. For GaN and AlN we get values of $\varepsilon_{\mathrm{o}}(\infty)=5.18$ and $\varepsilon_{\mathrm{e}}(\infty)=5.31$ as well as of $\varepsilon_{\mathrm{o}}(\infty)=4.13$ and $\varepsilon_{\mathrm{e}}(\infty)=$ 4.27 , respectively. A comparison with other results is reported in Ref. [52].

\subsection{Dielectric function of hexagonal InN}

Among the group-III nitride semiconductor compounds, the physical properties of $\mathrm{InN}$ are known rather poorly. This is mainly attributed to the difficulties 
in growing high-quality crystals. A discussion of optical properties of $\operatorname{In} \mathrm{N}$ demands to distinguish between either sputtered films or epitaxial layers deposited by MBE and MOVPE. Interband optical absorption measurements of sputtered polycrystalline InN films yielded a band gap of $E_{\mathrm{g}} \sim 1.9 \mathrm{eV}$ at room temperature [10]. This value of the band gap has been accepted for a long time and was frequently used as the end-point value for the extrapolation of the band gap in $\operatorname{In}_{x} \mathrm{Ga}_{1-x} \mathrm{~N}$ alloys. The wide use might surprise because Sullivan et al. reported an appreciably lower value of $E_{\mathrm{g}} \sim 1.7 \mathrm{eV}$ already in the late $80 \mathrm{~s}$ [54]. Recent studies of epitaxial layers grown by MBE [11, 55, 56] or MOVPE [57], however, revealed a much narrower gap of $E_{\mathrm{g}}<1 \mathrm{eV}$.

For both types of films, reports on the DF covering a broad energy range are scarce. Refractive index and extinction coefficient of sputtered layers were obtained from transmittance/reflectance [58] and SE [59] studies. However, the applied single-layer model neglects the influence of roughness and interface layers on the spectra. Guo et al. reported optical constants for MOVPE grown InN determined from reflectance investigations followed by Kramers-Kronig analysis. These data suffer from the relatively low InN layer thickness $(\sim 300 \mathrm{~nm})$ and the neglect of sample surface roughness [60].

Here, a comprehensive SE data analysis is presented based on multi-layer models in order to determine the InN DF's for both single-crystalline MBE grown and sputtered films with a high reliability. A single-crystalline hexagonal InN film was grown on (0001) sapphire by MBE as described elsewhere [61]. The sample consists of a $10 \mathrm{~nm}$ AlN nucleation layer followed by a $310 \mathrm{~nm}$ GaN buffer layer and the final $960 \mathrm{~nm}$ InN film. Structural properties of the films were examined by high resolution X-ray diffraction (HRXRD) measurements. The optical axis is oriented normal to the surface. From reciprocal space maps of the symmetric (002) and the asymmetric (20.5) Bragg reflexes the InN lattice constants were determined amounting to $c=5.686 \AA$ and $a=3.552 \AA$ which is in excellent agreement with previous studies of thick InN films deposited on GaN [62]. AFM revealed a surface roughness value of $\delta=12 \mathrm{~nm}$. Hall measurements at room temperature yielded an electron density and mobility of $8 \times 10^{17} \mathrm{~cm}^{-3}$ and $1500 \mathrm{~cm}^{2} /(\mathrm{V} \mathrm{s})$, respectively. The polycrystalline InN layers were deposited by magnetron sputtering of a nitrided indium target in a reactive nitrogen plasma [10] on Si substrates with a thickness of $1210 \mathrm{~nm}$, respectively. The lattice constants are $c=5.786 \AA$ and $a=3.58 \AA$ while the roughness amounts to $\delta \sim 8 \mathrm{~nm}$.

Figures $8 \mathrm{a}$ and b show the spectral dependence of $\Psi$ for the single-crystalline MBE film and the sputtered layer, respectively, taken at different angles of incidence (open circles). Remarkable differences are observed. For the sputtered layer in Fig. 8b, $\Psi$ shows oscillatory behaviour below $\sim 2 \mathrm{eV}$ due to interference, but a smooth dependence on the photon energy at higher energy. Obviously, the film is transparent in the low-energy range and strongly absorbing above $2 \mathrm{eV}$. In contrast, for the MBE grown film oscillations start to evolve only below $\sim 1 \mathrm{eV}$ 


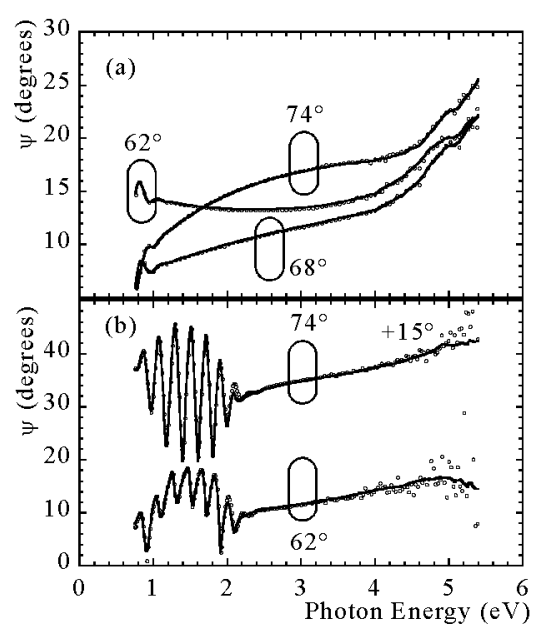

Fig. 8. Fit (full lines) of ellipsometric data (open circles) taken at different angles of incidence. Curves of (a) and (b) refer to a MBE-grown single-crystalline $\operatorname{InN}$ film and InN obtained by sputtering techniques, respectively. Data taken at $74^{\circ}$ in (b) are shifted by $15^{\circ}$ for the sake of clarity.

indicating a much smaller band gap for this film. It is worth noting that $\Delta$, not presented here, shows similar features. The full lines indicate the fit results taking into account the film roughness. No assumption were made concerning the shape of the DF, i.e. we fitted the values of $\varepsilon_{1}$ and $\varepsilon_{2}$ for each photon energy.

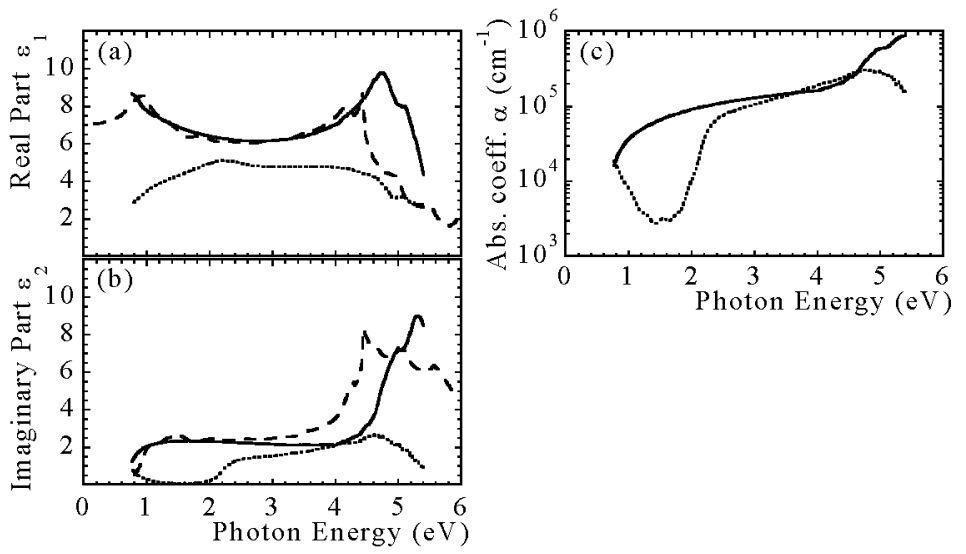

Fig. 9. Comparison of the real (a) and imaginary (b) part of the DF as well as the absorption coefficient (c) for hexagonal $\operatorname{InN}$. The full and dashed lines are obtained from the by point-by-point fits of the MBE-grown and sputtered layers, respectively. The long-dashed lines represent the results of the calculations for the ordinary component without quasi-particle corrections [28]. 
Figure 9 shows the results for the real (a) and imaginary (b) part of the DF for InN as well as the corresponding absorption coefficient (c). The full and dashed lines are obtained for the MBE grown and sputtered films, respectively. Similar to the GaN studies, the data can be assumed to represent nearly the ordinary DF. Calculations within the framework of density functional theory (DFT) in its local density approximation (LDA) yield for this polarisation direction the spectral dependence as indicated by the long-dashed lines [27]. Let us first focus on the absorption coefficient (c), however. Our results for the sputtered films are in consistency with previous investigations [10]. Those layers show only weak absorption in the energy range between 1 and $2 \mathrm{eV}$. For single-crystalline $\mathrm{InN}$, however, the absorption coefficient increases continuously with the photon energy.

The main result of this work is shown in Fig. 9b where the imaginary parts of the DF's are compared. The MBE grown films exhibit a spectral dependence of $\varepsilon_{2}$ which coincides in essential features with the theoretical results. Above the band gap $\left(E_{\mathrm{g}} \approx 0.75 \mathrm{eV}\right)$ the imaginary part increases very sharply indicating a non-parabolic band structure and remains nearly constant above the band gap up to energies of about $4 \mathrm{eV}$. The strong increase at higher energies arises from transitions along the $\mathrm{L}-\mathrm{M}$ direction in the Brillouin-zone and was also calculated in Refs. [24] and [63]. However, its energetic position depends on the assumptions made in the DFT-LDA procedure. For the sputtered films such a strong increase around $4 \mathrm{eV}$ is not observed, i.e. the two types of $\operatorname{InN}$ films differ not only in their band gap values but in addition in the higher-energetic parts of the DF. Therefore, we conclude that only the MBE grown films represent bulk-like $\operatorname{InN}$ in the wurtzite structure. Preliminary results of ellipsometry studies on $\operatorname{In}_{1-x} \mathrm{Ga}_{x} \mathrm{~N}$ films [12] emphasise this conclusion. As expected for an alloy system the spectral shape of both parts of the DF does not change very much if a low amount of Ga atoms replace In atoms in the InN lattice. With increasing Ga-content the step-like structure of $\varepsilon_{2}$ found between 1 and $2 \mathrm{eV}$ shifts continuously to higher energies reflecting the rising band gaps. But even for $43 \% \mathrm{Ga}$ composition, the gap energy is unambiguously below $1.9 \mathrm{eV}$, the value reported for sputtered films.

\subsection{Influence of electric fields on the dielectric function}

It is well accepted and widely used that electric fields changes the shape of the dielectric field. In a single particle picture the influence is described in terms of the Franz-Keldysh effect giving rise to pronounced oscillations above the band gap which allow e.g. the determination of electric field strength $F$ in a semiconductor. However, electron-hole interaction during the excitation process cannot be disregarded. It leads to bound states appearing in absorption measurements as strong peaks below the band gap as well as to an enhanced transition probability above the band gap known as the exciton continuum. Calculations showed that an electric field broadens the excitonic peak in the imaginary part of the DF and changes its energetic position in a nontrivial manner $[64,65]$. The characteristic field unit 
is the ionisation field strength $F_{i}$, for which the potential energy drop over one exciton Bohr radius corresponds to the exciton binding energy. For GaN a value of $F_{i}=81 \mathrm{kV} / \mathrm{cm}$ is estimated. Internal electric fields in nitride heterostructures exceeds this value appreciably due to spontaneous and piezoelectric polarisation effects. But even in within the surface space charge region $F>F_{i}$ is normally found.

Figure 10 shows photocurrent spectra of a Pt/GaN Schottky diode with $N$-face polarity taken at a temperature of $T=5 \mathrm{~K}$. Applying a positive forward bias of $0.5 \mathrm{~V}$ (full line) strong peaks due to the free excitonic transitions $F X^{A}, F X^{B}$, and $F X^{C}$ are clearly visible. In contrast the spectrum measured under a reverse bias of $-1 \mathrm{~V}$ (dashed line) does not show peaks any longer, however, in the low-energy range the absorption edge is strongly broadened. Obviously the field strength within the space charge region exceeds $F_{i}$ leading to an "exciton dead layer" (EDL).

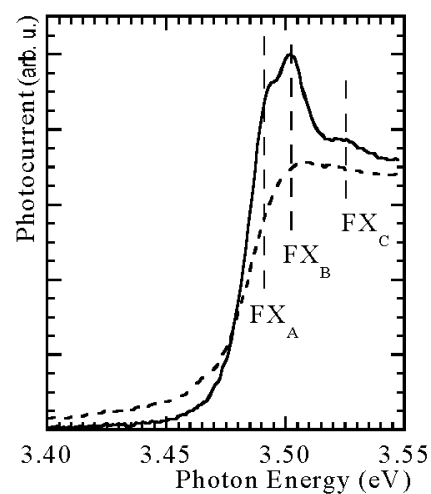

Fig. 10. Photocurrent spectra of a N-face Pt/ $\alpha-\mathrm{GaN}$ Schottky diode taken at a temperature of $T=5 \mathrm{~K}$ under applied forward and reverse bias voltages of $+0.5 \mathrm{~V}$ and $-1.0 \mathrm{~V}$, respectively.

In order to get a better insight into the general behaviour, the contribution of $\Gamma_{9}^{\mathrm{V}}-\Gamma_{7}^{\mathrm{c}}$ transitions to $\varepsilon_{2}$ was calculated as a function of $F$ in terms of $F_{i}$. Electron-hole interaction was included using the scheme developed by Blossey [65]. The results are given in Fig. $11 \mathrm{a}$, the dashed vertical line indicates the $F X^{A}$ transition energy at zero field strength. For low fields, the exciton ground state undergoes a shift to lower photon energies (Stark effect), reaching a minimum at a field of $F \approx 0.5 F_{i}$. Then it moves back towards higher energies and its width increases. For $F>F_{i}$, the ground state merges into the exciton continuum and, owing to the large broadening and the low amplitude, discrete excitonic features are no longer observable in the DF, implying the quenching of discrete exciton states. Above the band gap the DF shows as well oscillatory behaviour with an energetic spacing similar to the Franz-Keldysh oscillations within the single-particle picture. Once 
knowing the imaginary part the real part as presented in Fig. 11b is obtained by Kramers-Kronig transformation. Similar results are obtained for the two $\Gamma_{7}^{\mathrm{v}}-\Gamma_{7}^{\mathrm{c}}$ ( $F X^{B}$ and $F X^{C}$ ) transitions. Taking these data and adding the contribution of the higher energetic critical points one gets the complete DF under the influence of an electric field.
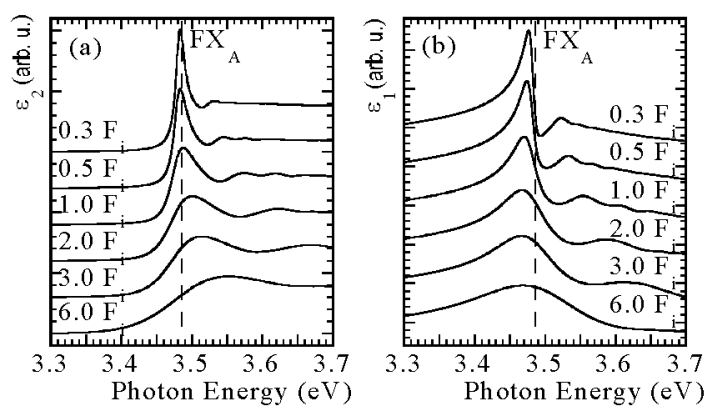

Fig. 11. Calculated contribution of $\Gamma_{9}^{\mathrm{v}}-\Gamma_{7}^{\mathrm{c}}$ transitions to the imaginary part (a) of the $\mathrm{DF}$ for $\alpha$-GaN for various electric field strengths at $T=5 \mathrm{~K}$. Electron-hole interaction (exciton effects) is taken into account. The corresponding real parts (b) are obtained via Kramers-Kronig transformation of $\varepsilon_{2}$.

The extension of the EDL depends on the space charge width, i.e. on the gate voltage. A spatial varying DF in this range causes interference effects in the reflectance. It can be evidenced by superimposing a modulation to the bias voltage while measuring the change in reflectance $(\Delta R)$ phase sensitively by a lock-in amplifier (electroreflectance). With increasing reverse bias $\Delta R$ shows a sign inversion proving interference effects. Such rotational spectra can analysed in order to determine the doping concentration or the surface barrier height as demonstrated recently [66].

However, the main results of this part is that that the exciton peak position does not only depend on the strain [8] but also on the electric field strength. In comparison to the field free case the largest deviation is found for $F \approx 0.5 F_{i}$, which amounts to $\sim 3.5 \mathrm{meV}$ for $F X^{A}$.

\section{Summary}

It was demonstrated that peculiarities of nitride samples such as roughness and interface layers require to establish appropriate multi-layer models for fitting spectroscopic ellipsometry or reflectance data in order to obtain reliable dielectric functions. Above the band gap only the ordinary component of the DF tensor was determined for hexagonal GaN as well as for InN. Anisotropy studies in this range require samples with $c$-axis orientation within the surface plane. But those studies 
have not been reported so far. For the latter compound a comparison of experimental data with calculations emphasises a band gap energy as low as $\sim 0.75 \mathrm{eV}$. Spectroscopic ellipsometry provides a very sensitive technique for determining the anisotropy below the band gap as the results for GaN and AIN showed. Electric fields alter the shape of the dielectric function around the band gap strongly. The Stark shift has to be taken into account when analysing exciton energies. Finally, the methods and models presented here concerning DF determination for the hexagonal phase of the nitrides are also applicable to the cubic counterparts.

\section{Acknowledgments}

I would like to thank my colleagues S. Shokhovets, D. Fuhrmann, G. Gobsch, V. Cimalla, and O. Ambacher from the TU Ilmenau for the fruitful collaboration and many stimulating discussions. I am indebted to W. Richter, V. Lebedev, A. Hangleiter, U. Rossow, W. Schaff, H. Lu, and K.S. Butcher for providing the samples. The DF of InN was calculated by F. Bechstedt and J. Furthmüller. Finally, I acknowledge financial support by the Thuringia Ministry of Science, Research, and Art under contract No. B609-02004.

\section{References}

[1] S. Nakamura, G. Fasol, The Blue Laser Diode, 1st ed., Springer-Verlag, Berlin 1988 , p. 1.

[2] N. Biyikli, I. Kimukin, T. Kartaloglu, O. Aytur, E. Ozbay, Appl. Phys. Lett. 82, 2344 (2003).

[3] L.F. Eastman, V. Tilak, V. Kaper, J. Smart, R. Thompson, B.M. Green, J.R. Shealy, T. Prunty, Phys. Status Solidi A 194, 433 (2002).

[4] D.J. As, A. Richter, J. Busch, M. Lübbers, J. Mimkes, K. Lischka, Appl. Phys. Lett. 76, $13(2000)$.

[5] Z.-F. Li, W. Lu, H.-J. Ye, Z.-H. Chen, X.-Z. Yuan, H.-F. Dou, S.-C. Shen, G. Li, S.J. Chua, J. Appl. Phys. 86, 2691 (1999).

[6] A. Kasic, M. Schubert, S. Einfeldt, D. Hommel, T.E. Tiwald, Phys. Rev. B 62, $7365(2000)$.

[7] W.R.L. Lambrecht, B. Segall, J. Rife, W.R. Hunter, D.K. Wickenden, Phys. Rev. $B \mathbf{5 1}, 13516$ (1995).

[8] B. Gil, in: Gallium Nitride (GaN) II, in series Semiconductors and Semimetals, Eds. J.I. Pankove, T.D. Moustakas, Vol. 57, Academic Press, San Diego 1999, p. 209.

[9] L.X. Benedict, T. Wethkamp, K. Wilmers, C. Cobet, N. Esser, E.L. Shirley, W. Richter, M. Cardona, Solid State Commun. 112, 129 (1999).

[10] T.L. Tansley, C.P. Foley, J. Appl. Phys. 59, 3241 (1986).

[11] T. Inushima, V.V. Mamutin, V.A. Vekshin, S.V. Ivanov, T. Sakon, M. Motokawa, S. Ohoya, J. Cryst. Growth 227-228, 481 (2001). 
[12] R. Goldhahn, S. Shokhovets, V. Cimalla, L. Spiess, G. Ecke, O. Ambacher, J. Furthmüller, F. Bechstedt, H. Lu, W.J. Schaff, Mat. Res. Soc. Symp. Proc. 743, L.5.9 (2003).

[13] M.J. Bergmann, H.C. Casey, Jr., J. Appl. Phys. 84, 1196 (1998).

[14] H.M. Ng, D. Doppaludpudi, E. Iliopoulos, T.D. Moustakas, Appl. Phys. Lett. 74, 1036 (1999).

[15] S. Shokhovets, R. Goldhahn, G. Gobsch, T.S. Cheng, C.T. Foxon, G.D. Kipshidze, Wo. Richter, J. Appl. Phys. 86, 2602 (1999).

[16] T. Schmidtling, M. Drago, U.W. Pohl, W. Richter, J. Cryst. Growth 248, 523 (2003).

[17] R. Goldhahn, S. Shokhovets; in: III-Nitride Semiconductors: Optical Properties $I I$, in series Optoelectronic Properties of Semiconductors and Superlattices, Eds. M.O. Manasreh, H.X. Jiang, Vol. 14, Taylor \& Francis Books, New York 2002, p. 73 .

[18] A.R.A. Zauner, M.A.C. Devillers, P.R. Hageman, P.K. Larsen, S. Porowski, MRS Internet J. Nitride Semicond. Res. 3, 17 (1998).

[19] U. Köhler, D.J. As, B. Schöttker, T. Frey, K. Lischka, J. Scheiner, S. Shokhovets, R. Goldhahn, J. Appl. Phys. 85, 404 (1999).

[20] Y. Hiroyama, M. Tamura, Jpn. J. Appl. Phys. Phys. 2, Lett. 37, L630 (1998).

[21] S. Ruvimov, Z. Lilienthal-Weber, J. Washburn, T.J. Drummond, M. Haifich, S.R. Lee, Appl. Phys. Lett. 71, 2931 (1997).

[22] S. Shokhovets, R. Goldhahn, V. Cimalla, T.S. Cheng, C.T. Foxon, J. Appl. Phys. 84, 1561 (1998).

[23] J.F. Nye, Physical Properties of Crystals, 1st ed., Oxford University Press, Oxford 1985 , p. 235.

[24] N.E. Christensen, I. Gorczyca, Phys. Rev. B 50, 4397 (1994).

[25] W.R.L. Lambrecht, S.N. Rashkeev, Phys. Status Solidi B 217, 599 (2000).

[26] L.X. Benedict, E.L. Shirley, Phys. Rev. B 59, 5441 (1999).

[27] F. Bechstedt, J. Furthmüller, M. Ferhat, L.K. Teles, L.M.R. Scolfaro, J.R. Leite, V.Yu. Davydov, O. Ambacher, R. Goldhahn, Phys. Status Solidi A 195, 628 (2003).

[28] K. Kornitzer, T. Ebner, K. Thonke, R. Sauer, C. Kirchner, V. Schwegler, M. Kamp, M. Leszczynski, I. Grzegory, S. Porowski, Phys. Rev. B 60, 1471 (1999).

[29] R.M.A. Azzam, N.M. Bashara, Ellipsometry and Polarized Light, 1st ed., Elsevier Science Publishers B.V. North Holland, Amsterdam 1989.

[30] M. Schubert, Phys. Rev. B 53, 4265 (1996); M. Schubert, B. Rheinländer, J.A. Woollam, B. Johs, C.M. Herzinger, J. Opt. Soc. Am. A 13, 875 (1996).

[31] R. Dingle, D.D. Sell, S.E. Stokowski, M. Ilegems, Phys. Rev. B 4, 1211 (1971).

[32] G. Yu, H. Ishikawa, T. Egawa, T. Soga, J. Watanabe, T. Jimbo, M. Umeno, Jpn. J. Appl. Phys. Phys. 2, Lett. 36, L1029 (1997).

[33] D.E. Aspnes, J. Opt. Soc. Am. 70, 1275 (1980). 
[34] C. Cobet, N. Esser, J.T. Zettler, W. Richter, P. Waltereit, O. Brandt, K.H. Ploog, S. Peters, N.V. Edwards, O.P.A. Lindquist, M. Cardona, Phys. Rev. B 64, 165203 (2001).

[35] N.V. Edwards, M.D. Bremser, T.W. Weeks, Jr., R.S. Kern, R.F. Davis, D.E. Aspnes, Appl. Phys. Lett. 69, 2065 (1996).

[36] T. Wethkamp, Optical Properties of Group-III-Nitrides in the Visible to VacuumUltraviolet Spectral Range Investigated by Spectroscopic Ellipsometry, 1st ed., Mensch \&Buch Verlag, Berlin 1999.

[37] S.W. King, J.P. Barnak, M.D. Bremser, K.M. Tracy, C. Ronning, R.F. Davis, R.J. Nemanich, J. Appl. Phys. 84, 5248 (1998).

[38] R. Goldhahn, S. Shokhovets, J. Scheiner, G. Gobsch, T.S. Cheng, C.T. Foxon, U. Kaiser, G.D. Kipshidze, W. Richter, Phys. Status Solidi A 177, 107 (2000).

[39] T. Kawashima, H. Yoshikawa, S. Adachi, S. Fuke, K. Ohtsuka, J. Appl. Phys. 82, 3528 (1997).

[40] D.V. Sivukhin, Zh. Eksp. Teor. Fiz. 30, 374 (1956) [Sov. Phys. JETP 3, 269 (1956)].

[41] D.E. Aspnes, J.B. Theeten, F. Hottier, Phys. Rev. B 20, 3292 (1979).

[42] D.E. Aspnes, Thin Solid Films 89, 249 (1982).

[43] J.C. Maxwell-Garnett, Philos. Trans. R. Soc. Lond. 203, 385 (1904); ibid. A 205, 237 (1906).

[44] D.A.G. Bruggeman, Ann. Physik (Leipzig) 24, 636 (1935).

[45] H.E. Bennett, J.O. Porteus, J. Opt. Soc. Am. 51, 123 (1961); J.O. Porteus, J. Opt. Soc. Am. 53, 1394 (1963); H.E. Bennett, Opt. Eng. 17, 480 (1978).

[46] I. Ohlidal, K. Navratil, F. Lukes, J. Opt. Soc. Am. 61, 1630 (1971); I. Ohlidal, K. Navratil, F. Lukes, Surf. Sci. 45, 91 (1974).

[47] L.T. Romano, in: Gallium Nitride and Related Semiconductors, EMIS Datareviews Series, Eds. by J.H. Edgar, S. Strite, I. Akasaki, H. Amano, C. Wetzel, Vol. 23, INSPEC, London 1999, p. 239

[48] N. Wieser, M. Klose, F. Scholz, J. Off, Y. Dutrieux, Mat. Sci. Forum 264-268, 1355 (1998).

[49] S. Shokhovets, R. Goldhahn, T.S. Cheng, C.T. Foxon, Semicond. Sci. Technol. 14, 181 (1999).

[50] H. Amano, N. Watanabe, N. Koide, I. Akasaki, Jpn. J. Appl. Phys. Phys. D, Let. 32, L1000 (1993).

[51] M.J. Bergmann, Ü. Özgür, H.C. Casey, Jr., H.O. Everitt, J.F. Muth, Appl. Phys. Lett. 75, 67 (1999).

[52] S. Shokhovets, R. Goldhahn, G. Gobsch, S. Piekh, R. Lantier, A. Rizzi, V. Lebedev, W. Richter, J. Appl. Phys. 94, 307 (2003).

[53] J. Pastrňák, L. Roskovcov, Phys. Status Solidi 14, K5 (1966); L. Roskovcová, J. Pastrňák, R. Babušková, Phys. Status Solidi 20, K29 (1967).

[54] B.T. Sullivan, R.R. Parsons, K.L. Westra, M.J. Brett, J. Appl. Phys. 64, 4144 (1988). 
[55] V. Yu. Davydov, A.A. Klochikhin, R.P. Seisyan, V.V. Emtsev, S.V. Ivanov, F. Bechstedt, J. Furthmüller, H. Harima, A.V. Mudryi, J. Aderhold, O. Semchinova, J. Graul, Phys. Status Solidi B 229, R1 (2002).

[56] J. Wu, W. Walukiewicz, K.M. Yu, J.W. Ager III, E.E. Haller, H. Lu, W.J. Schaff, Y. Saito, Y. Nanishi, Appl. Phys. Lett. 80, 3967 (2002).

[57] T. Matsuoka, H. Okamoto, M. Nakao, H. Harima, E. Kurimoto, Appl. Phys. Lett. 81, 1246 (2002).

[58] R.T. Shamrell, C. Parman, Opt. Mater. 13, 289 (1999).

[59] F. Li, D. Mo, C.B. Cao, Y.L. Zhang, H.L. Chan, C.L. Choy, J. Mat. Sci., Mat. Electr. 12, 725 (2001).

[60] Q. Guo, O. Kato, M. Fujisawa, A. Yoshida, Solid State Commun. 83, 721 (1992); Q. Guo, H. Ogawa, A. Yoshida, J. Electron Spectrosc. Relat. Phenom. 79, 9 (1996).

[61] H. Lu, W.J. Schaff, J. Hwang, H. Wu, G. Koley, L.F. Eastman, Appl. Phys. Lett. 79, 1489 (2001).

[62] S. Yamaguchi, M. Kariya, S. Nitta, T. Takeuchi, C. Wetzel, H. Amano, I. Akasaki, J. Appl. Phys. 85, 7682 (1999).

[63] C. Persson, R. Ahuja, A. Ferreira da Silva, B. Johansson, J. Phys., Condens. Matter 13, 8945 (2001).

[64] J.D. Dow, D. Redfield, Phys. Rev. B 1, 3358 (1970).

[65] D.F. Blossey, Phys. Rev. B 2, 3976 (1970); D.F. Blossey, Phys. Rev. B 3, 1382 (1971)

[66] S. Shokhovets, D. Fuhrmann, R. Goldhahn, G. Gobsch, O. Ambacher, M. Hermann, U. Karrer, M. Eickhoff, Appl. Phys. Lett. 82, 1712 (2003). 